\title{
Free-Space Squeezing Assists Perfectly Matched Layers in Simulations on a Tight
} Domain

Shyroki, Dzmitry; Ivinskaya, Aliaksandra; Lavrinenko, Andrei

Published in:

I E E E Antennas and Wireless Propagation Letters

Link to article, DOI:

10.1109/LAWP.2010.2049250

Publication date:

2010

Document Version

Publisher's PDF, also known as Version of record

Link back to DTU Orbit

Citation (APA):

Shyroki, D., Ivinskaya, A., \& Lavrinenko, A. (2010). Free-Space Squeezing Assists Perfectly Matched Layers in Simulations on a Tight Domain. I E E E Antennas and Wireless Propagation Letters, 9, 389-392.

https://doi.org/10.1109/LAWP.2010.2049250

\section{General rights}

Copyright and moral rights for the publications made accessible in the public portal are retained by the authors and/or other copyright owners and it is a condition of accessing publications that users recognise and abide by the legal requirements associated with these rights.

- Users may download and print one copy of any publication from the public portal for the purpose of private study or research.

- You may not further distribute the material or use it for any profit-making activity or commercial gain

- You may freely distribute the URL identifying the publication in the public portal 


\title{
Free-Space Squeezing Assists Perfectly Matched Layers in Simulations on a Tight Domain
}

\author{
Dzmitry M. Shyroki, Aliaksandra M. Ivinskaya, and Andrei V. Lavrinenko
}

\begin{abstract}
To minimize computer memory consumption in the finite-difference modeling, one tends to place computational domain boundaries as close to the simulated object as possible. Unfortunately, this leads to inaccurate solution in the case when evanescent electromagnetic field is expected to spread far outside the object, as in simulations of eigenmodes or scattering at a wavelength comparable to or larger than the object itself. Here, we show how, in addition to applying the perfectly matched layers (PMLs), outer free space can be squeezed to avoid cutting the evanescent field tails by the PMLs or computational domain borders. Adding the squeezetransform layers to the standard PMLs requires no changes to the finite-difference algorithms.
\end{abstract}

Index Terms-Coordinate transformation, finite-difference frequency-domain (FDFD) method, perfectly matched layer (PML).

\section{INTRODUCTION}

$\mathbf{P}$ ERFECTLY matched layers (PMLs) are the standard tool of choice when absorbing boundary conditions in the finite-difference or finite-element simulations are to be imposed [1]. In their unsplit version introduced originally in [2], [3], they can be derived via coordinate transformation $x \mapsto x^{\prime}$ : $x=\int_{0}^{x^{\prime}} s_{(1)}\left(x^{\prime}, \omega\right) \mathrm{d} x^{\prime}$ along the direction in which the outgoing radiation should be absorbed, with the complex "coordinate-stretching" function $s_{(1)}=\partial x / \partial x^{\prime}$

$$
s_{(1)}\left(x^{\prime}, \omega\right)=1+\frac{\sigma_{(1)}\left(x^{\prime}\right)}{i \omega}
$$

in its simplest form. Here, $x^{\prime}$ is a real-valued "computational" coordinate, necessarily bound: $x^{\prime} \in[-b, b] ; \sigma_{(1)}\left(x^{\prime}\right)$ is the PML "conductivity" profile - normally polynomial over an interval $[a, b]$, assuming that the PML starts at $x=a ; \omega$ is the lightwave frequency; the $\mathrm{e}^{i \omega t}$ convention for the time dependence is adopted. The layers built via (1) are known to be insufficient, however, when the evanescent fields are present [4], [5], and a common recipe is to modify (1) as

$$
s_{(2)}\left(x^{\prime}, \omega\right)=\xi\left(x^{\prime}\right)+\frac{\sigma_{(2)}\left(x^{\prime}\right)}{i \omega}
$$

Manuscript received April 08, 2010; accepted April 22, 2010. Date of publication April 29, 2010; date of current version May 10, 2010.

D. M. Shyroki is with the Institute of Optics, Information and Photonics, University of Erlangen-Nürnberg, 91054 Erlangen, Germany, and also with the Max Planck Institute for the Science of Light, 91054 Erlangen, Germany (e-mail: dzmitry.shyroki@mpl.mpg.de).

A. M. Ivinskaya and A. V. Lavrinenko are with the Department of Photonics Engineering, Technical University of Denmark, 2800 Kongens Lyngby, Denmark (e-mails: aliv@ fotonik.dtu.dk; alav@fotonik.dtu.dk).

Digital Object Identifier 10.1109/LAWP.2010.2049250 with the real-axis mapping function $\xi\left(x^{\prime}\right)>1$ over the PML interval $[a, b]$, and $\xi\left(x^{\prime}\right)=1$ otherwise. Nontrivial choice for the $\xi\left(x^{\prime}\right)$ function amounts to coordinate stretching or squeezing in the $x$-direction; to see that, we interpret (2) as the inverse jacobian of two consequential mappings: one giving standard PMLs via (1), and another, $x^{\prime} \mapsto x^{\prime \prime}$, with the real-valued derivative $\partial x^{\prime} / \partial x^{\prime \prime}=\xi\left(x^{\prime \prime}\right)$

$$
s_{(2)}\left(x^{\prime \prime}, \omega\right)=\frac{\partial x}{\partial x^{\prime}} \frac{\partial x^{\prime}}{\partial x^{\prime \prime}}=\xi\left(x^{\prime \prime}\right)+\frac{\sigma_{(1)}\left(x^{\prime}\left(x^{\prime \prime}\right)\right) \xi\left(x^{\prime \prime}\right)}{i \omega} .
$$

Such two-stage construction suggests one could first place standard PMLs (1) far enough from the simulated object, and then squeeze the outer space (both the PMLs and the space between the PMLs and the object) according to [6] and [7] in order to save computer time and memory. This would lead to absorbing boundaries exactly as defined by (2), except for the region of nontrivial $\xi$ being wider than that of nonzero PML conductivity $\sigma$. It is surprising that although the coordinate-stretching nature of (1) and (2) has been recognized by many previous authors [8]-[11], we are unaware of attempts to employ the above two-stage construction. Below, we show that by doing this very simple trick-that is, choosing a wider interval (say, two or three times the PML width) for the real-space squeezing function $\xi$-considerable accuracy improvement in simulations involving evanescent fields is achieved. This choice of wider region of nontrivial $\xi$ is much more important in getting improved numeric results than any optimization of the shape of $\xi$.

\section{FORMULATION}

In the following, we use the wavelength $\lambda$ instead of the frequency $\omega$, and a standard optimized expression for $\sigma\left(x^{\prime}\right)$ so that (1) reads

$$
s_{(1)}=1-i \sigma_{\max }\left(\frac{\left|x^{\prime}\right|-a^{\prime}}{b^{\prime}-a^{\prime}}\right)^{p}
$$

with the dimensionless constant

$$
\sigma_{\max }=20 \frac{p+1}{4 \pi} \frac{\lambda}{b^{\prime}-a^{\prime}}
$$

where the factor of 20, chosen rather arbitrarily, defines the logarithmic damping by an idealized (continuous) PML; $p$ is the PML conductivity profile order, 2 being a common choice. Upon further space squeezing $x^{\prime} \mapsto x^{\prime \prime}$, $\partial x^{\prime} / \partial x^{\prime \prime} \equiv \xi\left(x^{\prime \prime}\right)$, each quantity having the dimension of length is transformed to the double-primed squeezed coordinates in which actual computations are done; in particular, 


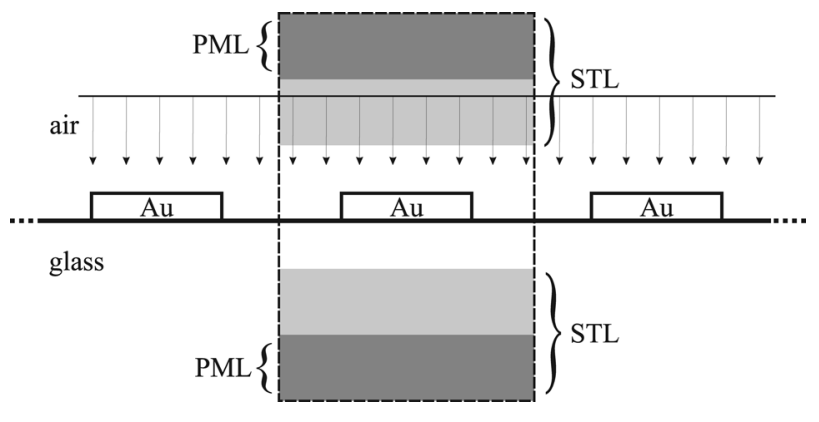

Fig. 1. 1D Au grating simulated on a 2D domain with periodic boundaries on the left and right, and PMLs + STLs below and above the grating.

$\lambda^{\prime \prime}\left(x^{\prime \prime}\right)=\lambda \partial x^{\prime \prime} / \partial x^{\prime}=\lambda / \xi\left(x^{\prime \prime}\right)$. Therefore, the new "stretching factor" $s_{(2)}\left(x^{\prime \prime}\right)=\xi\left(x^{\prime \prime}\right) s_{(1)}\left(x^{\prime}\left(x^{\prime \prime}\right)\right)$ becomes

$$
s_{(2)}=\xi-i \sigma_{\max }\left(\frac{\left|x^{\prime \prime}\right|-a^{\prime \prime}}{b^{\prime \prime}-a^{\prime \prime}}\right)^{p}
$$

where, similarly to (5)

$$
\sigma_{\max }=10 \frac{p+1}{4 \pi} \frac{\lambda}{b^{\prime \prime}-a^{\prime \prime}} .
$$

The real-valued squeezing function in (6) can be chosen as, for example, $\xi\left(x^{\prime \prime}\right)=\cos ^{-2}\left[\pi / 2\left(\left|x^{\prime \prime}\right|-\underline{a}^{\prime \prime}\right) /\left(b^{\prime \prime}-\underline{a}^{\prime \prime}\right)\right]$ on $\left[\underline{a}^{\prime \prime}, b^{\prime \prime}\right]$, and 1 otherwise, which corresponds to the arctan-mapping of $\left[\underline{a}^{\prime}, \infty\left[\right.\right.$ onto the $\left[\underline{a}^{\prime \prime}, b^{\prime \prime}\left[\right.\right.$ interval, $\underline{a}^{\prime \prime}=\underline{a}^{\prime}$. Further construction of the boundary layer by modifying the dielectric and magnetic constants $\epsilon$ and $\mu$ within $\left[\underline{a}^{\prime \prime}, b^{\prime \prime}\right.$ is straightforward [7]. If initially, in the primed coordinates, the PML extends from $a^{\prime}>\underline{a}^{\prime}$ to $b^{\prime}=\infty$, then after squeezing, in the double-primed coordinates, the PML is totally contained within thus defined squeeze-transform layer (STL), the former being "wider" than the PML. Normally, we employed STLs starting immediately from the boundaries of an object under study. A differentiable function other than arctan - for example, tanh — can be used to construct STLs, but this affects numeric results much less than the choice of the widths of the PML and STL layers, and hence will not be considered in the following.

\section{EXAMPLES}

First, we simulate light scattering from a periodic one-dimensional (1D) grating of rectangular gold bars deposited on a glass substrate as shown in Fig. 1. The grating period is $750 \mathrm{~nm}$, and the bars are $20 \mathrm{~nm}$ high and $112 \mathrm{~nm}$ wide, as in [12]. Localized plasmon resonances are excited in this system in the $p$ polarization, as indicated by extinction peak between 600 and $700 \mathrm{~nm}$ (its position is angle- and period-independent) seen in Fig. 2. Each curve is obtained for normally incident $p$ wave with the full-vector two-dimensional (2D) finite-difference frequencydomain (FDFD) method on a computational domain discretized into $1.2 \times 1.2 \mathrm{~nm}^{2}$ pixels with periodic boundaries to the left and to the right and standard PMLs above and below the grating, each of the two composed of 10 sublayers. One sees that increasing the domain size in the direction normal to the grating from 120 to $480 \mathrm{~nm}$ (and the overall resolution from $100 \times 625$ to $400 \times 625$ pixels) is vital to obtain a smooth, well-converged

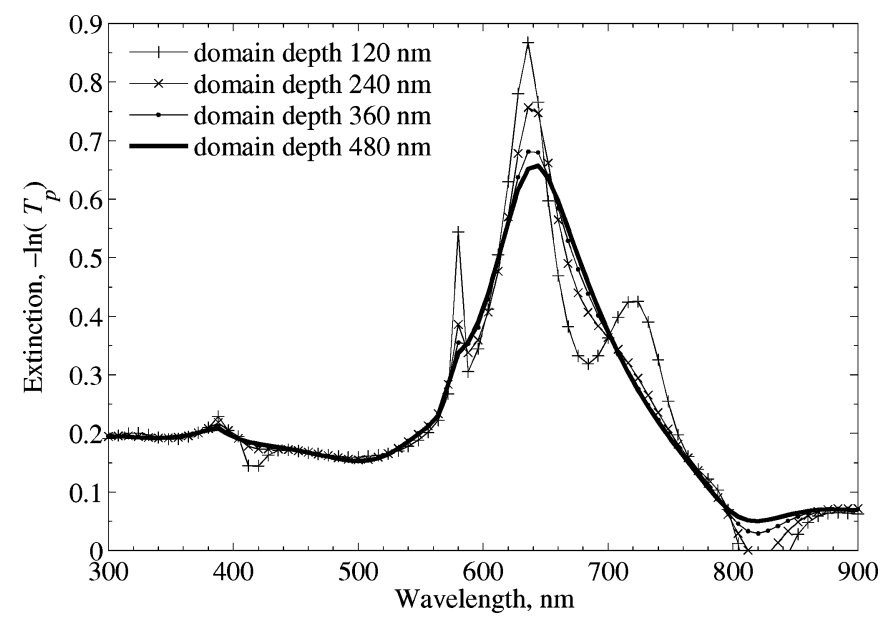

Fig. 2. Sensitivity of the $p$-wave extinction of an array of the $20 \times 112 \mathrm{~nm}^{2}$ gold stripes (as shown in Fig. 1) to the computational domain "depth," i.e., its vertical dimension where standard PMLs are applied.
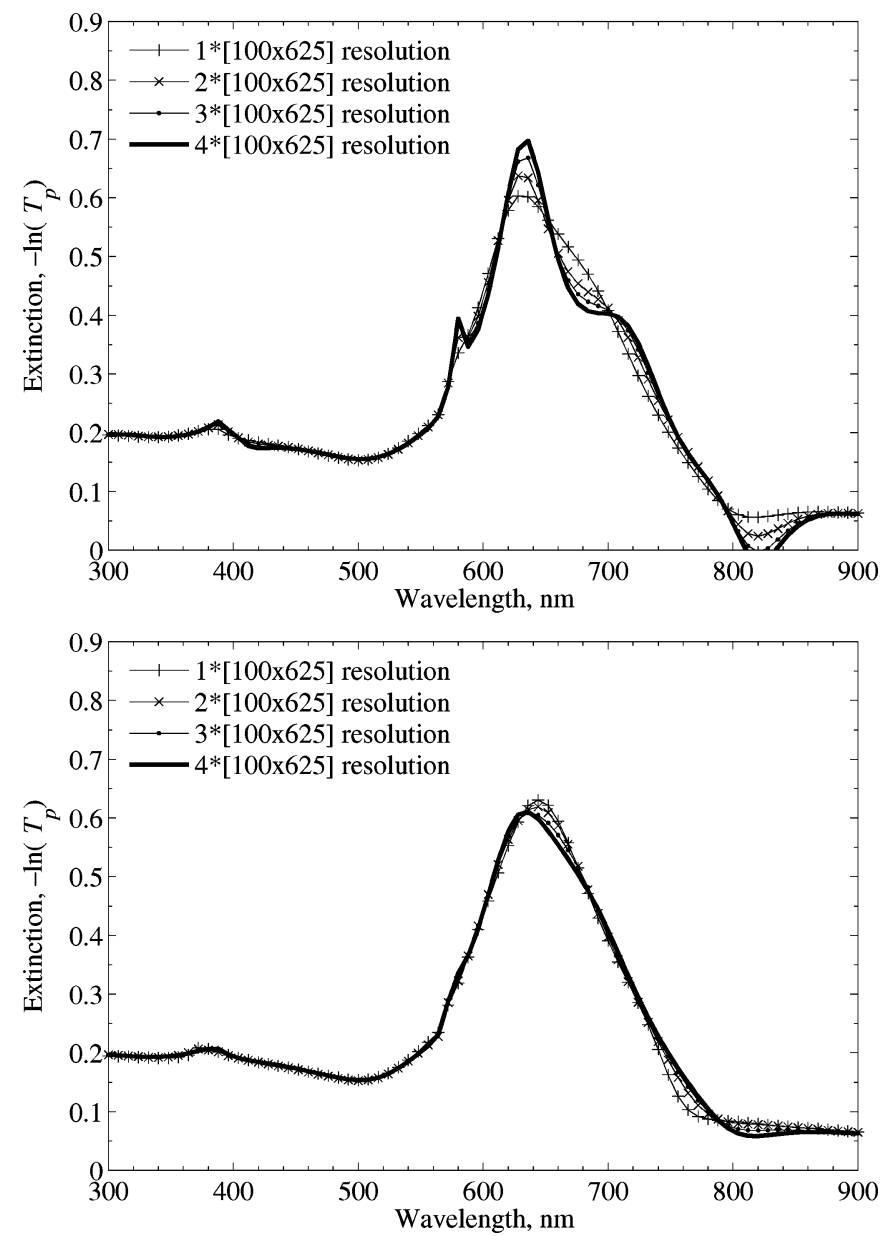

Fig. 3. Sensitivity of the spectrum as in Fig. 2 to the resolution in the $120 \times 750 \mathrm{~nm}^{2}$ computational window, for the PMLs combined with the double- (upper figure) and triple-wide (lower figure) STLs.

spectrum. In Fig. 3, we repeat the same simulations on a $120 \times 750 \mathrm{~nm}^{2}$ domain again with 10-sublayer PMLs, but now adding the STLs of double (20 sublayers) and triple (30 sublayers) width. We see that with triple-wide STLs, the spectrum 


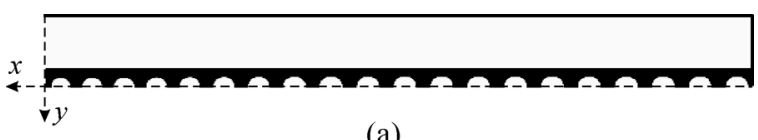

(a)

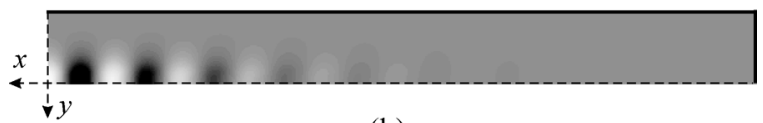

(b)

Fig. 4. (a) Dielectric permittivity profile of the quarter of a nanobeam as in [13]. (b) Distribution of the $E_{z}$ field of the $\mathrm{TM}_{00}$ eigenmode.

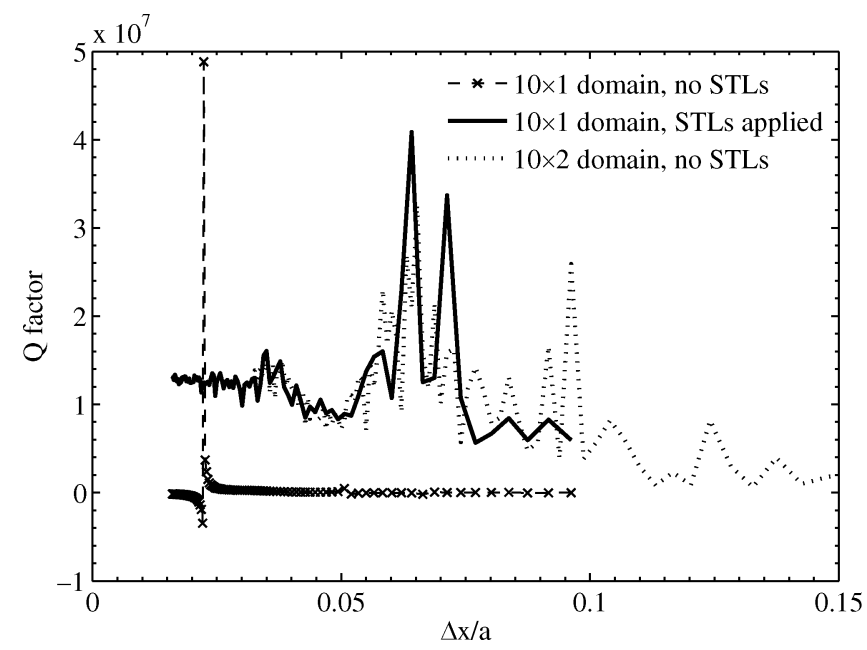

Fig. 5. $\mathrm{TM}_{00}$ mode quality factor versus pixel size $\Delta x(=\Delta y)$, obtained on a $10 \times 1$ computational domain with and without STLs applied and on the doublewide $(10 \times 2)$ domain without STLs.

is free from anomalies and well-converged, complying with the bold curve in Fig. 2 obtained on the $480 \times 750 \mathrm{~nm}^{2}$ domain, and with the results of modeling with the Fourier modal method [12]. Similar improvements by adding three-times-wider STLs over standard PMLs were observed in calculated spectra of many other 1D and 2D metal gratings.

Our second example is eigenmode analysis of a photonic crystal nanobeam cavity made of a silicon $(n=3.4)$ plate in air with 1D array of holes as described in [13] and shown in Fig. 4, where only a quarter of the system is present. Given the half-width of the plate $0.5 a$, an individual hole segment length varies between $a_{1}=0.84 a$ and $a_{9 \ldots 20}=a$, and the corresponding hole diameter equals $0.6 a_{i}$. The length of the half of the plate (and of the computational window) is $\sum a_{i}=19.24 a$, and it is assumed sufficiently extended in the third direction, so it lends itself to the 2D FDFD modeling. The width of the computational domain was chosen as either $(1 / 10) \sum a_{i}=1.924 a$ (the " $10 \times 1$ "domain) or twice that, i.e., $3.849 a$ (the " $10 \times 2$ " domain). The PMLs occupied one-third of the free space between the plate and the computational window boundary, and the STLs (when applied additionally to the PMLs) covered all the free space. To find the $\mathrm{TM}_{00}$ mode with a maximum for $E_{z}$ in the center of coordinates, both the $x=0$ and $y=0$ mirror planes were mimicked by the perfect magnetic conductors.

Fig. 5 shows the quality factor $Q=\Re(k) /[2 \Im(k)]$ ( $k$ is the complex wavenumber obtained as a solution to Maxwell's equations represented in the frequency domain as an eigenproblem)

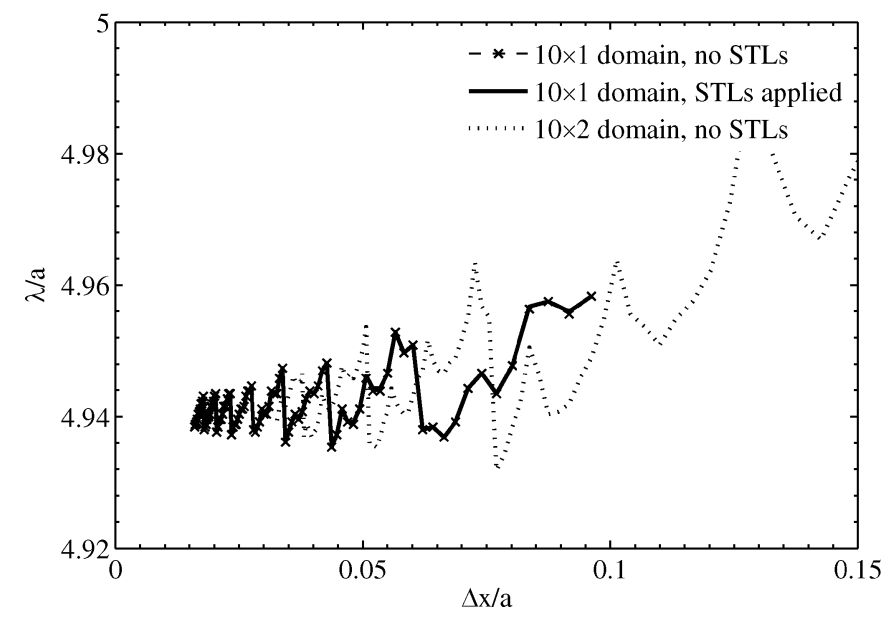

Fig. 6. Same as Fig. 5, but for the normalized eigenwavelength of the $\mathrm{TM}_{00}$ mode versus pixel size.

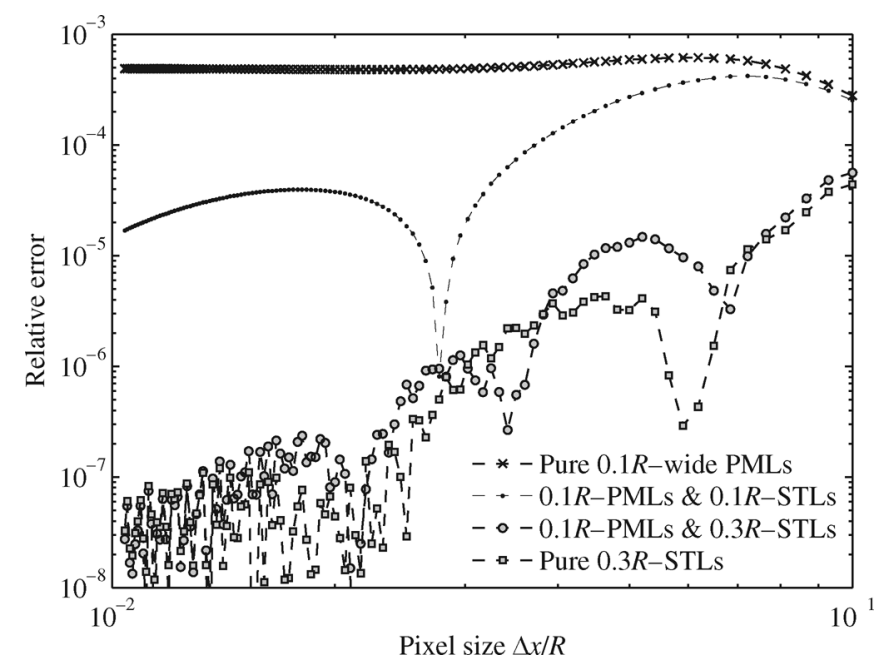

Fig. 7. Relative error $\left|\beta-\beta_{0}\right| / \beta_{0}$ in the fundamental mode propagation constant $\beta$ of a step-index fiber of radius $R$ and index contrast 1.5:1.515 calculated with the full-vector 2D FDFD method.

of the $\mathrm{TM}_{00}$ mode as a function of the pixel size $\Delta x(=\Delta y)$. We see that, on a narrow domain, pure PMLs are inadequate, but adding the STLs allows to get the same accuracy as with the standard PMLs on a twice wider domain (wide enough to provide stable $Q$ results without space squeezing). In Fig. 6, all the curves show similar convergence to the eigenwavelength $\lambda_{\mathrm{TM}}=4.94 a$, the two $10 \times 1$-curves now being almost indistinguishable. Of course, we remember that simulations on the wider domain require double computation resources, while adding STLs to PMLs on a narrow domain improves $Q$ factor accuracy at no cost.

For the third and final example, consider a guided mode in a conventional step-index fiber. In this case (assuming the fiber is straight, and the core-cladding interface perfectly smooth), no oscillatory waves propagate from the core of the fiber, but evanescent field tails spread outside the core, their decay rate at a given wavelength being proportional to the core-cladding index contrast. We have chosen a pretty low contrast fiber of $n_{\text {clad }}: n_{\text {core }}=1.5: 1.515$ and simulated its fundamental mode at a wavelength $0.5 R$ ( $R$ is the radius of the fiber) on 
a $1.3 R$-wide computation domain (for symmetry reasons, a quarter of the fiber can be modeled with magnetic and electric walls cutting the fiber through its center). At the chosen wavelength, the analytically calculated propagation constant is 1.508860106 (in the $\omega / c$ units), and the evanescent field tails decay slowly, so they are considerably disturbed by the boundaries of our $1.3 R$-wide computation domain. From Fig. 7 , we see that applying $0.1 R$-wide PMLs leads to a very poor accuracy of approximately $10^{-3}$, not improving with increasing the resolution (in fact, this is just slightly better than when no PMLs are present at all); adding the STLs of similar width does improve the accuracy by more than an order of magnitude and, furthermore, provides a slow but nonzero convergence with resolution. Nonetheless, it is only when we employ $0.3 R$-wide STLs with the same 0.1R-wide PMLs that the results become as good as those obtained with pure $0.3 R$-wide STLs. This is a great success because, in the absence of radiation fields, the PMLs are not actually needed at all, and pure STLs were shown to be the most adequate tool [6], [7].

\section{CONCLUSION}

A combination of PMLs with wider squeeze-transform layers (STLs) greatly improves the accuracy of electromagnetic modeling on a tight domain. We recommend the ratio between the PML and STL widths be 1:2 or 1:3 and STLs start right from the borders of a simulated object. Because coding the STLs is so simple and calculations with STLs require no extra computer resources except a tiny overhead when generating $\epsilon$ and $\mu$ on a preprocessing stage, adding STLs to PMLs should become a standard practice in the finite-difference time-domain (FDTD) and FDFD modeling.

\section{REFERENCES}

[1] A. Taflove, Computational Electrodynamics: The Finite-Difference Time-Domain Method. Boston, MA: Artech House, 2005.

[2] L. Zhao and A. C. Cangellaris, "GT-PML: Generalized theory of perfectly matched layers and its application to the reflectionless truncation of finite-difference time-domain grids," IEEE Trans. Microw. Theory Tech., vol. 44, no. 12, pt. 2, pp. 2555-2563, Dec. 1996.

[3] S. D. Gedney, "An anisotropic perfectly matched layer-absorbing medium for the truncation of FDTD lattices," IEEE Trans. Antennas Propag., vol. 44, no. 12, pp. 1630-1639, Dec. 1996.

[4] J. De Moerloose and M. A. Stuchly, "Behavior of Berenger's ABC for evanescent waves," IEEE Microw. Guided Wave Lett., vol. 5, no. 10, pp. 344-346, Oct. 1995.

[5] J. Fang and Z. Wu, "Generalized perfectly matched layer for the absorption of propagating and evanescent waves in lossless and lossy media," IEEE Trans. Microw. Theory Tech., vol. 44, no. 12, pt. 1, pp. 2216-2222, Dec. 1996.

[6] D. M. Shyroki, "Squeezing of open boundaries by Maxwell-consistent real coordinate transformation," IEEE Microw. Wireless Compon. Lett., vol. 16, no. 11, pp. 576-578, Nov. 2006.

[7] D. M. Shyroki and A. V. Lavrinenko, "Perfectly matched layer method in the finite-difference time-domain and frequency-domain calculations," Phys. Status Solidi B, vol. 244, no. 10, pp. 3506-3514, 2007.

[8] P. G. Petropoulos, "Reflectionless sponge layers as absorbing boundary conditions for the numerical solution of Maxwell's equations in rectangular, cylindrical and spherical coordinates," SIAM J. Appl. Math., vol. 60, no. 3, pp. 1037-1058, 2000.

[9] F. L. Teixeira and W. C. Chew, "General closed-form PML constitutive linear media," IEEE Microw. Guided Wave Lett., vol. 8, no. 6, pp. 223-225, Jun. 1998.

[10] J. P. Hugonin and P. Lalanne, "Perfectly matched layers as nonlinear coordinate transforms: A generalized formalization," J. Opt. Soc. Amer. A, vol. 22, pp. 1844-1849, 2005.

[11] J. Čtyroký, "Efficient boundary conditions for bidirectional propagation algorithm based on Fourier series," J. Lightw. Techn., vol. 27, no. 14, pp. 2575-2582, Jul. 15, 2009.

[12] N. L. Dmitruk, A. V. Korovin, O. I. Mayeva, and M. V. Sosnova, "Role of local plasmons in interaction of light with 1D periodic ensembles of metallic nanowires," Plasmonics, vol. 4, pp. 193-200.

[13] Y. Zhang, M. W. McCutcheon, I. B. Burgess, and M. Loncar, "Ultra-high-Q TE/TM dual-polarized photonic crystal nanocavities," Opt. Lett., vol. 34, no. 17, pp. 2694-2696, 2009. 